Bull. Austral. Math. Soc.

Vol. 51 (1995) [383-393]

\title{
SOME SERIES INVOLVING THE ZETA FUNCTION
}

\author{
Junesang Choi, H.M. SRivastava and J.R. Quine
}

Lots of formulas for series of zeta function have been developed in many ways. We show how we can apply the theory of the double gamma function, which has recently been revived according to the study of determinants of Laplacians, to evaluate some series involving the Riemann zeta function.

\section{INTRODUCTION}

An over two-century old theorem of Christian Goldbach (1690-1764), which was stated in a letter dated 1729 from Goldbach to Daniel Bernoulli (1700-1782), has recently been posed as the following:

Problem. (Shallit and Zikan [15]). Let $\mathcal{S}$ be the set of nontrivial integer $k$ th powers, that is,

$$
\mathcal{S}=\left\{n^{k} \mid n \geqslant 2, k \geqslant 2\right\}=\{4,8,9,16,25,32,36, \ldots\} .
$$

Show that

$$
\sum_{\omega \in \mathcal{S}}(\omega-1)^{-1}=1
$$

the sum being extended over all members $\omega$ of $\mathcal{S}$.

In terms of the Riemann zeta function (see Titchmarsh [18] and Ivić [10])

$$
\zeta(s)=\sum_{n=1}^{\infty} \frac{1}{n^{*}}=\frac{1}{1-2^{-s}} \sum_{n=1}^{\infty} \frac{1}{(2 n-1)^{s}} \quad(\operatorname{Re}(s)>1),
$$

the summation formula (1.2) becomes (see $[15, \mathrm{p} .403])$

$$
\sum_{k=2}^{\infty}\{\zeta(k)-1\}=1
$$

Received 6th June, 1994

This paper was supported in part by $\mathrm{N} 94123$ for the first author.

Copyright Clearance Centre, Inc. Serial-fee code: 0004-9729/95 \$A2.00+0.00. 
As a matter of fact, it is not difficult to show also that

$$
\begin{gathered}
\sum_{k=2}^{\infty}(-1)^{k}\{\zeta(k)-1\}=\frac{1}{2} \\
\sum_{k=2}^{\infty}\{\zeta(2 k)-1\}=\frac{3}{4} \text { and } \sum_{k=1}^{\infty}\{\zeta(2 k+1)-1\}=\frac{1}{4} .
\end{gathered}
$$

Formula (1.4) and hence also (1.2), and its variations (1.5) and (1.6) are, of course, equivalent to various (known or easily derivable) sums of double series. The object of this paper is to evaluate some series involving $\zeta(s)$ and the generalised (Hurwitz's) zeta function $\zeta(s, a)$ defined usually by (see $[14, \mathrm{p} .22]$ )

$$
\zeta(s, a)=\sum_{n=0}^{\infty} \frac{1}{(n+a)^{z}} \quad(\operatorname{Re}(s)>1 \quad a \neq 0,-1,-2, \ldots),
$$

using the theory of the double gamma function. Using these results we can also obtain the explicit form of the triple gamma function $\Gamma_{3}$.

It should be remarked in passing that both $\zeta(s)$ and $\zeta(s, a)$ are analytic functions everywhere in the complex $s$-plane except for a simple pole at $s=1$ with its residue 1.

The double gamma function had been defined and studied by Barnes $[3,4,5]$ and others about 1900, not appearing in the tables of the most well-known special functions, cited in the exercise by Whittaker and Watson [20, p.264]. Recently this function has been revived in to the study of determinants of Laplacians. Shintani [16] also uses this function to prove the classical Kronecker limit formula. Its $p$-adic analytic extention appeared in a formula of Casson Nogués [6] for the $L p$-adic functions at the point 0 .

Before Barnes, these functions had been introduced in a different form by Hölder [9], Alexeiewsky [2], Kinkelin [13].

Barnes [3] defines the double gamma function $\Gamma_{2}=1 / G$ satisfying each of the following properties:

(a) $G(z+1)=\Gamma(z) G(z), z \in \mathbf{C}$;

(b) $G(1)=1$;

(c) As $n \rightarrow \infty$,

$$
\begin{aligned}
\log G(z+n+2)= & \frac{n+1+z}{2} \log (2 \pi)+\left[\frac{n^{2}}{2}+n+\frac{5}{12}+\frac{z^{2}}{2}+(n+1) z\right] \log n \\
& -\frac{3 z^{2}}{4}-n-n z-\log A+\frac{1}{12}+O\left(\frac{1}{n}\right)
\end{aligned}
$$


where $\Gamma$ is the well-known gamma function and $A$ is called Glaisher's (or Kinkelin's) constant, defined by

(1.9) $\quad \log A=\lim _{N \rightarrow \infty}\left\{\log \left(1^{1} 2^{2} \cdots N^{N}\right)-\left(\frac{N^{2}}{2}+\frac{N}{2}+\frac{1}{12}\right) \log N+\frac{N^{2}}{4}\right\}$

the numerical value of $A$ being $1.282427130 \cdots$.

From this definition, Barnes deduced

$$
\begin{aligned}
\left\{\Gamma_{2}(z+1)\right\}^{-1} & =G(z+1) \\
& =(2 \pi)^{z / 2} e^{-\left[(1+\gamma) z^{2}+z\right] / 2} \prod_{k=1}^{\infty}\left(1+\frac{z}{k}\right)^{k} e^{-z+z^{2} / 2 k}
\end{aligned}
$$

where $\gamma$ denotes the Euler-Mascheroni constant given by

$$
\gamma=\lim _{n \rightarrow \infty}\left(1+\frac{1}{2}+\cdots+\frac{1}{n}-\log n\right) \cong 0.577215664 \ldots
$$

2. Evaluation of SOME SERIES IN VOlving THE zeta FunCtion We first introduce Alexeiwsky's theorem [3]:

$$
\int_{0}^{z} \log \Gamma(1+t) d t=z \log \Gamma(z+1)-\frac{z(z+1)}{2}+\frac{z}{2} \log (2 \pi)-\log G(z+1) .
$$

Setting $z=1$ in (2.1), we obtain

$$
\int_{0}^{1} \log \Gamma(1+t) d t=\frac{1}{2} \log (2 \pi)-1
$$

Replacing $t+1$ by $t+a$ in the expression (1.10) for $G(t+1)$ and differentiating logarithmically the resulting formula with respect to $t$, we obtain

$$
\frac{d}{d t} \log G(t+a)=\frac{1}{2} \log (2 \pi)-\left(t+a-\frac{1}{2}\right)+(t+a-1) \frac{d}{d t} \log \Gamma(t+a) .
$$

Integrating both sides of (2.3) from $t=0$ to $t=z$, we find

$$
\begin{aligned}
\int_{0}^{z} \log \Gamma(t+a) d t= & \frac{z}{2} \log (2 \pi)-\frac{z(z+2 a-1)}{2} \\
& +(z+a-1) \log \Gamma(z+a)-\log \frac{G(z+a)}{G(a)}-(a-1) \log \Gamma(a),
\end{aligned}
$$


which is the natural extension of Alexeiewsky's theorem.

It is known [3] that

$$
\int_{0}^{1} \log G(t+a) d t=a \log \Gamma(a)-\log G(a+1)-\frac{a(a-1)}{2}+\frac{a}{2} \log (2 \pi)+C,
$$

where $C$ is a constant whose value is given by

$$
C=\frac{1}{4} \log \left(\frac{e^{1 / 3}}{2 \pi A^{8}}\right) .
$$

Indeed, let $f(a)=\int_{0}^{1} \log G(t+a) d t$. Differentiating $f(a)$ with respect to $a$ and considering $G(1+a)=\Gamma(a) G(a)$, we obtain $\frac{d}{d a} f(a)=\log \Gamma(a)$. It also follows from (2.4) that

$$
\int_{0}^{a} \log \Gamma(t) d t=a \log \Gamma(a)-\log G(a+1)-\frac{a(a-1)}{2}+\frac{a}{2} \log (2 \pi) .
$$

We thus obtain

$$
\int_{0}^{1} \log G(t+a) d t=a \log \Gamma(a)-\log G(a+1)-\frac{a(a-1)}{2}+\frac{a}{2} \log (2 \pi)+C,
$$

where $C$ is a constant. Putting $a=1$ in the resulting formula, we get

$$
C=\int_{0}^{1} \log G(t+1) d t-\frac{1}{2} \log (2 \pi)
$$

We thus have from (1.10) that

$$
\begin{aligned}
\int_{0}^{1} \log G(t+1) d t= & \frac{1}{4} \log (2 \pi)-\frac{5}{12}-\frac{\gamma}{6} \\
& +\sum_{k=1}^{\infty}\left[k(k+1) \log \left(1+\frac{1}{k}\right)-k-\frac{1}{2}+\frac{1}{6 k}\right] \\
= & \frac{1}{4} \log (2 \pi)-\frac{5}{12}-\frac{\gamma}{6} \\
& +\lim _{n \rightarrow \infty}\left[-2 \log A-\left(n^{2}+n+\frac{1}{6}\right) \log n+\frac{n^{2}}{2}\right. \\
& \left.\left(n^{2}+n\right) \log n+\frac{1}{2}-\frac{n^{2}}{2}+\frac{\gamma}{6}+\frac{1}{6} \log n\right] \\
= & \frac{1}{4} \log (2 \pi)+\frac{1}{12}-2 \log A
\end{aligned}
$$


from which we can derive the desired constant $C$ in (2.5).

Now it follows from $([17$, p.18] that (see Whittaker and Watson $[20$, p.276]; see also Gradshteyn and Ryzhik [8, p.1074], Entry 9. 532)

$$
\sum_{k=2}^{\infty}(-1)^{k} \zeta(k, a) \frac{t^{k}}{k}=\log \Gamma(a+t)-\log \Gamma(a)-t \psi(a) \quad(|t|<|a|)
$$

where $\psi(t)=\Gamma^{\prime}(t) / \Gamma(t)$ is often called the digamma function. Replacing $t$ by $-t$ in (2.8), adding both sides of the resulting equation and (2.8), respectively, we obtain

$$
\sum_{k=1}^{\infty} \zeta(2 k, a) \frac{t^{2 k}}{k}=\log \Gamma(a+t)+\log \Gamma(a-t)-2 \log \Gamma(a) \quad(|t|<|a|)
$$

Differentiating both sides of (2.9) with respect to $t$, and multiplying both sides of the resulting equation by $t^{2}$, we obtain

$$
2 \sum_{k=1}^{\infty} \zeta(2 k, a) t^{2 k+1}=t^{2} \psi(a+t)-t^{2} \psi(a-t) \quad(|t|<|a|)
$$

Integrating both sides of (2.10) from $t=0$ to $t=z$, we get

$$
\sum_{k=1}^{\infty} \zeta(2 k, a) \frac{t^{2 k+2}}{k+1}=\int_{0}^{z} t^{2} \psi(a+t) d t+\int_{0}^{-z} t^{2} \psi(a+t) d t \quad(|z|<|a|)
$$

Integrating by parts the first integral of the right-hand side of (2.11) successively, and evaluating the resulting integral by means of (2.3), we can derive

$$
\begin{aligned}
\int_{0}^{z} t^{2} \psi(a+t) d t= & z^{2} \log \Gamma(a+z)-2 \int_{0}^{z} t \log \Gamma(a+t) d t \\
= & -\left\{\frac{z^{2}}{4}+\frac{(1-a) z}{2}\right\} \log (2 \pi)+\frac{z^{3}}{3}+\frac{z^{2}}{4}-\frac{(a-1)(2 a-1) z}{2} \\
& +(a-1)^{2} \log \Gamma(z+a)+(z-a+1) \log G(z+a) \\
& +(a-1) \log G(a)-(a-1)^{2} \log \Gamma(a)-\int_{0}^{z} \log G(t+a) d t .
\end{aligned}
$$

Replacing $z$ by $-z$ in (2.12), and considering the resulting equation, (2.11) and (2.12), 
we can deduce that, for $|z|<|a|$,

$$
\begin{aligned}
\sum_{k=1}^{\infty} \zeta(2 k, a) \frac{z^{2 k+2}}{k+1}= & -\frac{z^{2}}{2} \log (2 \pi)+\frac{z^{2}}{2} \\
& +(a-1)^{2}\{\log \Gamma(a+z)+\log \Gamma(a-z)\} \\
& +(1-a)\{\log G(a+z)+\log G(a-z)\} \\
& +(a-1) \log G(a)-(a-1)^{2} \log \Gamma(a) \\
& -\int_{0}^{z} \log G(t+a) d t-\int_{0}^{-z} \log G(t+a) d t
\end{aligned}
$$

Setting $a=2$ in (2.13), we deduce that, for $|z|<2$

$$
\begin{aligned}
\sum_{k=1}^{\infty} \frac{\zeta(2 k)-1}{k+1} z^{2 k+2}= & -\frac{z^{2}}{2} \log (2 \pi)+\frac{z^{2}}{2} \\
& +\log \Gamma(2+z)+\log \Gamma(2-z) \\
& -\log G(2+z)-\log G(2-z) \\
& -\int_{0}^{z} \log G(t+2) d t-\int_{0}^{-z} \log G(t+2) d t .
\end{aligned}
$$

Letting $z=1$ in (2.14), we obtain

$$
\begin{aligned}
\sum_{k=1}^{\infty} \frac{\zeta(2 k)-1}{k+1}= & -\frac{1}{2} \log (2 \pi)+\frac{1}{2}+\log 2 \\
& -\int_{0}^{1} \log G(t+2) d t+\int_{0}^{1} \log G(t+1) d t .
\end{aligned}
$$

Setting $a=1$ and $a=2$ in (2.5), respectively, we obtain

$$
\begin{aligned}
& \int_{0}^{1} \log G(t+1) d t=\frac{1}{2} \log (2 \pi)+C, \\
& \int_{0}^{1} \log G(t+2) d t=\log (2 \pi)-1+C,
\end{aligned}
$$

where $C$ is the given constant in (2.5).

Finally, we can obtain summation formulas involving series of the zeta function from (2.15) and (2.16):

$$
\sum_{k=1}^{\infty} \frac{\zeta(2 k)-1}{k+1}=\frac{3}{2}-\log \pi
$$


Note. The formula (2.17) was evaluated in a different way in [7] and we showed there that the evaluation of the formula (2.17) is equivalent to that of the determinant of Laplacian on $\mathbf{S}^{\mathbf{3}}$.

In a similar way, we can derive

$$
\sum_{k=1}^{\infty} \frac{\zeta(2 k+1)-1}{2 k+3}=\frac{13}{12}-\frac{\gamma}{3}-\frac{1}{2} \log 2-2 \log A
$$

or, equivalently

$$
\log A=\frac{13}{24}-\frac{\gamma}{6}-\frac{1}{4} \log 2-\frac{1}{2} \sum_{k=1}^{\infty} \frac{\zeta(2 k+1)-1}{2 k+3}
$$

Indeed, replacing $t$ by $-t$ in (2.8), and subtracting (2.8) from the resulting equation, we obtain

$$
2 \sum_{k=1}^{\infty} \frac{\zeta(2 k+1, a)}{2 k+1} t^{2 k+1}=\log \Gamma(a-t)-\log \Gamma(a+t)+2 t \psi(a) \quad(|t|<|a|) .
$$

Differentiating both sides of (2.20) with respect to $t$, and multiplying both sides of the resulting equation by $t^{2}$, and next integrating both sides of the resulting equation from $t=0$ to $t=z$, and finally evaluating the resulting integrals by means of (2.12), we find that, for $|z|<|a|$,

$$
\begin{aligned}
2 \sum_{k=1}^{\infty} \frac{\zeta(2 k+1, a)}{2 k+3} z^{2 k+3}= & (1-a) z \log (2 \pi)+\frac{2}{3}(\psi(a)-1) z^{3}+(a-1)(2 a-1) z \\
& +(a-1)^{2}\{\log \Gamma(a-z)-\log \Gamma(a+z)\} \\
& -(z+a-1) \log G(a-z)-(z-a+1) \log G(a+z) \\
& +\int_{0}^{z} \log G(a+t) d t-\int_{0}^{-z} \log G(a+t) d t
\end{aligned}
$$

Setting $a=2$ in (2.21), and letting $z=1$ in the resulting equation and considering $\psi(2)=1-\gamma$ and (2.16), we can readily see the desired equation (2.18).

REMARK. It follows from [17, Equation (5.9)] that

$$
\sum_{k=1}^{\infty} \frac{\zeta(2 k+1)-1}{k+1}=\log 2-\gamma
$$


In the theory of the $\Gamma$-function, it is fairly well known (see for example, Jordan [12, p.62], Equation (2)) that

$$
\log \Gamma(1+t)=-\gamma t+\sum_{k=2}^{\infty}(-1)^{k} \zeta(k) \frac{t^{k}}{k} \quad(|t|<1)
$$

or, equivalently, (see, Abramowitz and Stegun [1, p.256, Equation (6.1.33)])

$$
\log \Gamma(2+t)=(1-\gamma) t+\sum_{k=2}^{\infty}(-1)^{k}(\zeta(k)-1) \frac{t^{k}}{k} \quad(|t|<2)
$$

By differentiating both sides of (2.23) with respect to $t$, we obtain (see for example, Jordan [12, p.327, Equation (2)])

$$
\psi(1+t)=-\gamma+\sum_{k=2}^{\infty}(-1)^{k} \zeta(k) t^{k-1} \quad(|t|<1)
$$

Multiplying both sides of (2.25) by $t^{2}$, and integrating both sides of the resulting equation from $t=0$ to $t=z$, we obtain

$$
\sum_{k=2}^{\infty}(-1)^{k} \zeta(k) \frac{z^{k+2}}{k+2}=\frac{\gamma}{3} z^{3}+\int_{0}^{z} t^{2} \psi(1+t) d t \quad(|z|<1)
$$

Setting $a=1$ in (2.12), and applying the resulting equation to (2.26), we have that, for $|z|<1$,

$$
\begin{aligned}
\sum_{k=2}^{\infty}(-1)^{k} \zeta(k) \frac{z^{k+2}}{k+2}=\frac{1}{4} & (1-\log (2 \pi)) z^{2}+\frac{1}{3}(1+\gamma) z^{3} \\
& +z \log G(z+1)-\int_{0}^{z} \log G(1+t) d t .
\end{aligned}
$$

It is readily seen from (2.7) and $G(2)=1$ with $z \rightarrow 1$ in (2.27) that

$$
\sum_{k=2}^{\infty}(-1)^{k} \frac{\zeta(k)}{k+2}=\frac{1}{2}-\frac{1}{2} \log (2 \pi)+\frac{\gamma}{3}+2 \log A
$$

It is noted that $[17$, p.13, Equation (5.5)]

$$
\sum_{k=2}^{\infty}(-1)^{k} \frac{\zeta(k)}{k+1}=1+\frac{1}{2} \gamma-\frac{1}{2} \log (2 \pi) \text {. }
$$


It follows from (2.24) in a similar manner that, for $|z|<2$,

$$
\begin{aligned}
\sum_{k=2}^{\infty}(-1)^{k}(\zeta(k)-1) & \frac{z^{k+2}}{k+2}=\left(\frac{z}{2}-\frac{z^{2}}{4}\right) \log (2 \pi)+\frac{\gamma}{3} z^{3}+\frac{z^{2}}{4}-\frac{3}{2} z \\
+ & \log \Gamma(z+2)+(z-1) \log G(z+2)-\int_{0}^{z} \log G(2+t) d t .
\end{aligned}
$$

Setting $z=1$ in (2.30), and considering (2.16), we obtain

$$
\sum_{k=2}^{\infty}(-1)^{k} \frac{\zeta(k)-1}{k+2}=-\frac{1}{2} \log \left(\frac{\pi}{2}\right)+\frac{\gamma}{3}-\frac{1}{3}+2 \log A .
$$

Vignéras $\left[19\right.$, p.241] introduces $n$-ple gamma functions $\Gamma_{n}$ by a recurrence formula. So we can readily evaluate the Weierstrass canonical product form of the triple gamma function $\Gamma_{3}$ more explicitly by applying the above results. We obtained $\Gamma_{3}$ as follows [7]:

$$
\begin{aligned}
& \Gamma_{3}(1+x)=G_{3}(1+x)= \\
& \quad \exp \left[-\frac{1}{6}\left(\gamma+\frac{\pi^{2}}{6}+\frac{3}{2}\right) x^{3}+\frac{1}{4}\left(\gamma+\log (2 \pi)+\frac{1}{2}\right) x^{2}+\Omega x\right] \\
& \quad \times \prod_{m \in \mathbb{N}^{2} \times N^{*}}\left(1+\frac{x}{L(m)}\right)^{-1} \exp \left[\frac{x}{L(m)}-\frac{1}{2}\left(\frac{x}{L(m)}\right)^{2}+\frac{1}{3}\left(\frac{x}{L(m)}\right)^{3}\right]
\end{aligned}
$$

where

$$
\Omega=\frac{1}{12}\left(\frac{3}{2}-\gamma-3 \log (2 \pi)+\frac{\pi^{2}}{12}\right)+\frac{1}{2} \sum_{n=1}^{\infty}(-1)^{n} \frac{1}{(n+3)(n+4)} \zeta(n+2),
$$

$L(m)=m_{1}+m_{2}+m_{3}$ if $m=\left(m_{1}, m_{2}, m_{3}\right) \in \mathbf{N}^{2} \times N^{*}, \mathbf{N}$ is the set of nonnegative integers and $\mathbf{N}^{*}=\mathbf{N}-\{0\}$.

Now $\Omega$ can be expressed in a closed form by means of some of the previous results. Indeed, it is readily seen from (2.28) and (2.29) with $\zeta(2)=\pi^{2} / 6$ that the summation part in $\Omega$ is

$$
\begin{aligned}
\sum_{k=3}^{\infty}(-1)^{k} \frac{1}{(k+1)(k+2)} \zeta(k) & =\sum_{k=3}^{\infty}(-1)^{k} \frac{\zeta(k)}{k+1}-\sum_{k=3}^{\infty}(-1)^{k} \frac{\zeta(k)}{k+2} \\
& =\frac{1}{2}+\frac{\gamma}{6}-\frac{\pi^{2}}{72}-2 \log A .
\end{aligned}
$$

We thus have

$$
\Omega=\frac{3}{8}-\frac{1}{4} \log (2 \pi)-\log A .
$$

NOTE. The explicit form of the triple gamma function $\Gamma_{3}$ is necessary to compute the determinant of Laplacian on $\mathbf{S}^{3}$, where $\mathbf{S}^{3}$ is the unit 3-sphere (see [7]). 


\section{REFERENCES}

[1] M. Abramowitz and I.A. Stegun, Handbook of mathematical functions (Dover Publications, Inc., 1965).

[2] W. Alexejewsky, 'Ueber eine Classe von Functionen, die der Gammafunction analog sind', Leipzig: Weidmanncshe Buchhandluns 46 (1894), 268-275.

[3] E.W. Barnes, 'The theory of G-function', Quart. J. Math. 31 (1899), 264-314.

[4] E.W. Barnes, 'Genesis of the double gamma function', Proc. London Math. Soc. 31 (1900), 358-381.

[5] E.W. Barnes, 'The theory of the double Gamma function', Philos. Trans. Roy. London Soc. Ser. A 186 (1901), 265-388.

[6] P. Cassou-Nogués, Analogues p-adiques des fonctions $\Gamma$-multiples (Journées Arithmétiques de Marseille, 1978).

[7] Junesang Choi, 'Determinant of Laplacian on $\mathbf{S}^{3}$, Math. Japon. 40 (1994), 155-166.

[8] I.S. Gradshteyn and I.M. Ryzhik, Tables of integrals, series and products, (Corrected and enlarged edition prepared by A. Jeffrey) (Academic Press, 1980).

[9] V.O. Hölder, 'Ueber eine transcendente function', Gottingen Dieterichsche VerlagsBuchhandlung (1886), 514-522.

[10] A. Ivić, The Riemann zeta-function (John Wiley and Sons, 1985).

[11] W.W. Johnson, 'Note on the numerical transcendents $S_{n}$ and $s_{n}=S_{n}-1$ ', Bull. Amer. Math. Soc. 12 (1905-1906), 477-482.

[12] C. Jordan, Calculus of finite differences (Chelsea Publishing Company, 1965).

[13] Kinkelin, 'Ueber eine mit der Gamma Function verwante Transcendente und deren Anwendung auf die Integralrechnung', J. Reine Angew. Math. 57 (1860), 122-158.

[14] W. Magnus, F. Oberhettinger and R.P. Soni, Formulas and theorems for the special functions of mathematical physics (Springer-Verlag, Berlin, Heidelberg, New York, 1966).

[15] J.D. Shallit and K. Zikan, 'A theorem of Goldbach', Amer. Math. Monthly 93 (1986), 402-403.

[16] T. Shintani, 'A proof of the classical Kronecker limit formula', Tokyo J. Math. 3 (1980), 191-199.

[17] H.M. Srivastava, 'A unified presentation of certain classes of series of the Riemann zeta function', Riv. Mat. Univ. Parma 14 (1988), 1-23.

[18] E.C. Titchmarsh, The theory of the Riemann zeta function (Clarendon Press, Oxford, 1951).

[19] M.F. Vignéras, 'L'Equation Fonctionnelle de la Fonction Zeta de Selberg du Groupe Modulaire PSL(2,Z)', Soc. Math. France Asté 61 (1979), 235-249.

[20] E.T. Whittaker and G.N. Watson, $A$ course of modern analysis (Cambridge University Press, 1963). 
Department of Mathematics Dongguk University

Kyongju 780714

Korea

Department of Mathematics Florida State University

Tallahassee FL 32304

United States of America
Department of Mathematics and Statistics University of Victoria

Victoria BC V8W 3P4

Canada 